\title{
Crystal structure of a new benzoic acid inhibitor of influenza neuraminidase bound with a new tilt induced by overpacking subsite $\mathrm{C} 6$
}

Lalitha Venkatramani ${ }^{1}$, Eric S Johnson², Gundurao Kolavi², Gillian M Air ${ }^{1}$, Wayne J Brouillette ${ }^{2^{*}}$ and Blaine HM Mooers ${ }^{1 *}$

\begin{abstract}
Background: Influenza neuraminidase (NA) is an important target for antiviral inhibitors since its active site is highly conserved such that inhibitors can be cross-reactive against multiple types and subtypes of influenza. Here, we discuss the crystal structure of neuraminidase subtype N9 complexed with a new benzoic acid based inhibitor (2) that was designed to add contacts by overpacking one side of the active site pocket. Inhibitor 2 uses benzoic acid to mimic the pyranose ring, a bis-(hydroxymethyl)-substituted 2-pyrrolidinone ring in place of the $\mathrm{N}$-acetyl group of the sialic acid, and a branched aliphatic structure to fill the sialic acid C6 subsite.

Results: Inhibitor 2 \{4-[2,2-bis(hydroxymethyl)-5-oxo-pyrrolidin-1-yl]-3-[(dipropylamino)methyl)]benzoic acid\} was soaked into crystals of neuraminidase of A/tern/Australia/G70c/75 (N9), and the structure refined with $1.55 \AA$ X-ray data. The benzene ring of the inhibitor tilted $8.9^{\circ}$ compared to the previous compound (1), and the number of contacts, including hydrogen bonds, increased. However, the $\mathrm{IC}_{50}$ for compound 2 remained in the low micromolar range, likely because one propyl group was disordered. In this high-resolution structure of NA isolated from virus grown in chicken eggs, we found electron density for additional sugar units on the N-linked glycans compared to previous neuraminidase structures. In particular, seven mannoses and two $\mathrm{N}$-acetylglucosamines are visible in the glycan attached to Asn200. This long, branched high-mannose glycan makes significant contacts with the neighboring subunit.

Conclusions: We designed inhibitor 2 with an extended substituent at C4-corresponding to C6 of sialic acid-to increase the contact surface in the C6-subsite and to force the benzene ring to tilt to maximize these interactions while retaining the interactions of the carboxylate and the pyrolidinone substituents. The crystal structure at $1.55 \AA$ showed that we partially succeeded in that the ring in 2 is tilted relative to 1 and the number of contacts increased, but one hydrophobic branch makes no contacts, perhaps explaining why the $I C_{50}$ did not decrease. Future design efforts will include branches of unequal length so that both branches may be accommodated in the C6-subsite without conformational disorder. The high-mannose glycan attached to Asn200 makes several intersubunit contacts and appears to stabilize the tetramer.
\end{abstract}

Keywords: Influenza neuraminidase inhibitor, Enzyme-ligand complex, Antiviral, Structure-based drug design, Glycoprotein, Glycan structure, Influenza virus, Benzoic acid, Pyrrolidinone

\footnotetext{
* Correspondence: wbrou@uab.edu; blaine-mooers@ouhsc.edu

1Department of Biochemistry and Molecular Biology, University of Oklahoma

Health Sciences Center, 941 Stanton L. Young Blvd, Oklahoma City, OK 73104,

USA

${ }^{2}$ Department of Chemistry and Center for Biophysical Sciences and

Engineering, University of Alabama at Birmingham, 901 14th Street South,

Birmingham, AL 35294, USA
}

\section{Biomed Central}

C 2012 Venkatramani et al.; licensee BioMed Central Ltd. This is an Open Access article distributed under the terms of the Creative Commons Attribution License (http://creativecommons.org/licenses/by/2.0), which permits unrestricted use, distribution, and reproduction in any medium, provided the original work is properly cited. 


\section{Background}

Influenza A viruses display two membrane-anchored glycoproteins, hemagglutinin (HA) and neuraminidase (NA). HA mediates attachment of the virus to sialic acid receptors on host cells to initiate virus infection. After virus replication, NA removes sialic acid residues from viral and cellular glycoproteins to facilitate virus release and allow spread of infection to new cells. In the absence of NA activity, the progeny virions aggregate and infection ends $[1,2]$.

The distinct antigenic properties of HA and NA from different viruses are used to classify influenza type A into subtypes-16 for HA and 9 for NA. H1N1 (1918), H2N2 (1957) and H3N2 (1968) caused the major influenza pandemics of the $20^{\text {th }}$ century. Evidence that the new HA and sometimes NA genes originated in wild birds before appearing in humans has raised concerns about the recent spread of highly pathogenic avian H5N1 viruses. These viruses have the potential to gain transmissibility among humans and thereby devastate immunologically naïve human populations [3]. Type B viruses are not carried by birds and are not divided into antigenic subtypes, but since the mid-1980s, two lineages, B/Victoria and B/Yamagata, have been evolving concurrently in humans [4].

NA is a good target for structure-based enzyme inhibitor design because its well-characterized active site is conserved across all influenza A and B viruses [5]. The 11 amino acids in the active site that interact with sialic acid remain unchanged amidst extensive genetic variation in the rest of the sequence [6-8]; for an alignment, see [9]. Structure-based drug design led to the successful antiviral drugs zanamivir (GG167) [10] and oseltamivir (GS4071) [11] that are effective against different types and subtypes of influenza. Zanamivir is based on a transition state analogue of sialic acid while oseltamivir replaces sialic acid's pyranose ring with a cyclohexene scaffold (Figure 1). The high potency and oral activity of oseltamivir encourages the use of alternative scaffolds to replace the sugar ring of sialic acid [12-14]. Benzene ring scaffolds minimize the number of chiral centers, potentially simplifying the chemical synthesis and reducing associated expenses [15-18]. The challenge is to configure substituents on the ring to optimize the interactions with the active site. The direct contacts with sialic acid are conserved in all influenza NAs, but the subsites that surround the sialic acid-named according to the ring atoms of sialic acid (Figure 1E)-show differences in size and shape between strains and sometimes account for large differences in potency between N1, N2 and B NAs $[17,19]$. In addition, inhibitors with a hydrophobic side chain often show differences in potency between the two structural groups of type A NA: group 1 (subtypes N1, N4, N5 and N8) and group 2 (subtypes N2, N3, N6, N7, and N9) [20].

$\mathrm{X}$-ray structures of several simple benzoic acid derivatives bound to influenza B NA show that these inhibitors bind to the active site in the same orientation as sialic acid $[15,16]$. The derivatives' common carboxylate substitution at the $\mathrm{C} 2$ position maintains the native interaction with the arginine triad (Arg292, Arg118, and Arg371 in $\mathrm{N} 2$ numbering), and the $\mathrm{N}$-acetyl substitution at the $\mathrm{C} 5$ subsite maintains the native interaction with Arg152.

In addition, using a benzene ring scaffold, hydrophobic groups of varying lengths fit into the glycerol binding site

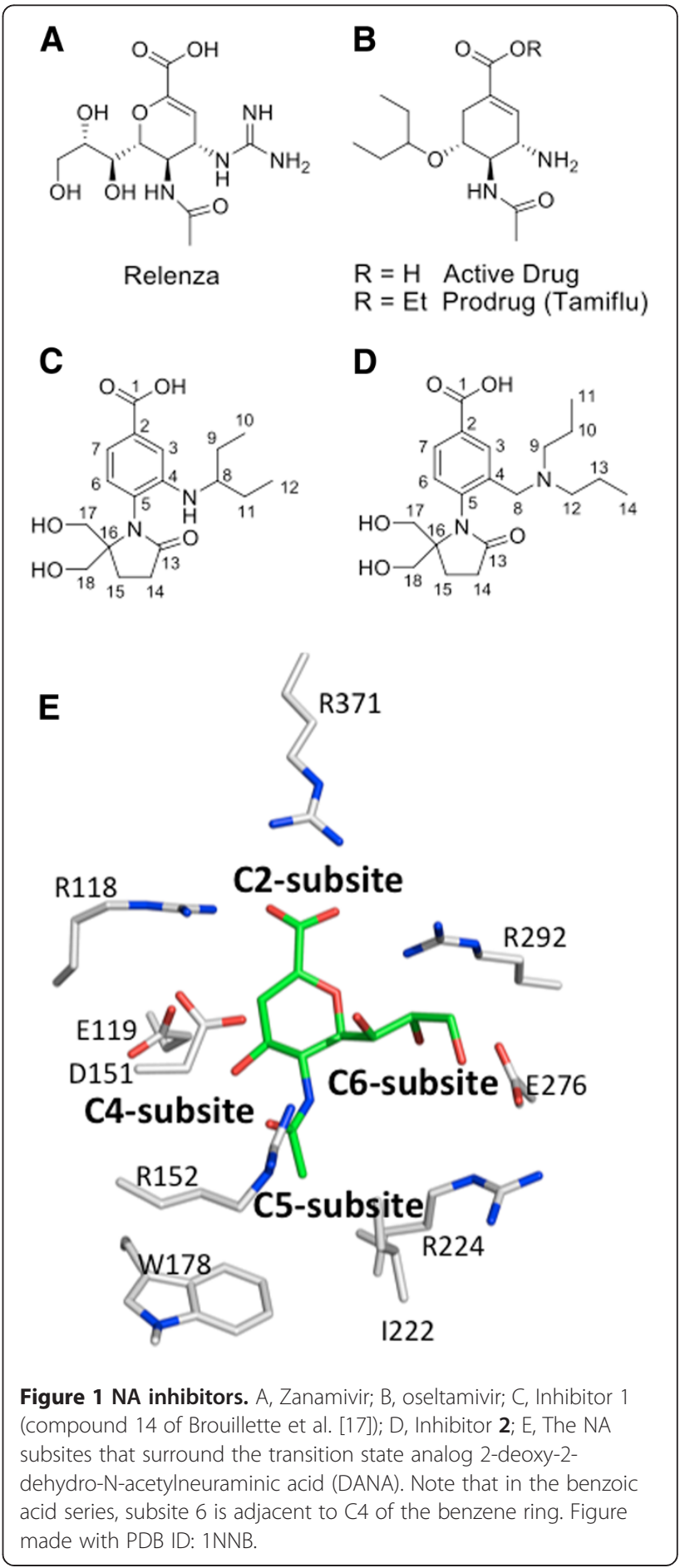


(C6 subsite) of the sialic acid [21]. The length and branching of the aliphatic chain have been modified to improve inhibition, but benzoic acids from this structural class have yet to match the inhibitory activities observed for zanamivir and oseltamivir. It is likely that this results from the lack of a basic group (aliphatic amine or guanidine) in the C4 subsite, which forms an important salt bridge with inhibitors in clinical use.

One of the best compounds from the existing benzoic acid series, inhibitor 1, (Figure $1 \mathrm{C}$ ) lacks the $\mathrm{C} 4$ subsite substituent yet exhibited moderately potent (low to mid $\mathrm{nM}$ ) activity against NAs of the N2 and N9 subtypes of influenza A virus, with less inhibitory activity against influenza B virus NA, as observed for other compounds with a similar hydrophobic substituent on the benzene ring [18].

We are trying to broaden the specificity of the benzoic acids against influenza viruses of different subtypes and to increase potency. The best way to accomplish this goal is likely by adding a basic substituent to form a salt bridge in the C4 subsite. However, attempts to design substituents on the benzene ring that occupy both the negatively charged $\mathrm{C4}$ subsite and the glycerol binding site (C6 subsite) of sialic acid have been difficult because the two subsites are offset from the plane of the benzene ring. The results of molecular modeling studies using FlexX [22] suggested that a different "tilt" of the benzene ring may be needed, and one way to alter the tilt may be to change the size of the hydrophobic substituent, such that an increase or a decrease in steric crowding reorients the benzene ring in the binding site.

The inhibitor studied here, compound $\mathbf{2}$, like compound $\mathbf{1}$, contains no chiral centers but has a longer hydrophobic substituent consisting of a 3-heptyl (instead of 3-pentyl) group positioned one atom further from the benzene ring (Figure 1D). Enzyme inhibition assays indicate that the affinity of $\mathbf{2}$ is in the low micromolar range for type A NAs but, compared to $\mathbf{1}$, lower for type B NA (Table 1) [12,17]. We have determined the crystal structure of 2 in complex with a type A N9 NA to a resolution of $1.55 \AA$, and we discuss it in terms of the successful reorientation of the benzene ring and the lack of improvement in binding affinity. The structure of the complex suggests routes to design inhibitors that might show improved affinity.

Table 1 IC $_{50}$ values of the inhibitor 2 compared with compound 1

\begin{tabular}{lll}
\hline Inhibitor & NA & IC50 $(\boldsymbol{\mu M})$ \\
\hline $\mathbf{1}[17]$ & A/Udorn/72 NA (N2) & 0.49 \\
& A/tern/Australia/G70c NA (N9) & 4.4 \\
& B/Lee/40 NA $[23]$ & 271 \\
& H3N2 & 2 \\
& H1N1 & 20 \\
& A/tern/Australia/G70c NA (N9) & $9.1 \pm 2.2$ \\
& B/Lee/40 NA & 180 \\
\hline
\end{tabular}

\section{Results and discussion}

\section{Overall structure}

N9 NA crystals that had been soaked with inhibitor 2 showed similar unit cell dimensions and space group symmetry as low temperature crystal structures of other N9-inhibitor complexes and the uncomplexed mutant R292K (Protein Data Bank identifier [PDB ID] 2QWA) [24]. The available uncomplexed native structures (PDB ID, 1NNA and 7NN9) were determined at room temperature and were not as suitable for direct comparison with our low temperature data, so we judged the crystal structure of the uncomplexed mutant R292K to be a less biased starting model for molecular replacement. Inhibitor $\mathbf{2}$ bound to the active site with nearly full occupancy (refined occupancy factor of 0.68) and did not bind to additional sites such as the second sialic acid binding site [25,26] (PDB ID, 2C4A).

\section{Inhibitor-N9 interactions}

The X-ray data were of high quality and the structure of 2 complexed with N9 NA was refined with $1.55 \AA$ X-ray data. The final model had good geometry (Table 2) with all amino acid residues in the allowed region of the Ramachandran plot $(96.1 \%$ in the favored region and $0 \%$ outliers). Initial $\mathrm{F}_{\mathrm{o}}-\mathrm{F}_{\mathrm{c}}$ maps showed clear electron density of the inhibitor in the active site of NA (Figure 2A). The two substituents of the inhibitor's pyrrolidine ring were buried inside the active site cavity (dihedral angle C6-C5-N5-C13 at the atropisomeric center $-112^{\circ}$ ) with no indication of alternative conformers with rotation about the $\mathrm{C} 8 \mathrm{-N}$ bond. The $F_{o}-F_{c}$ maps revealed very good electron density for the propyl group involving $\mathrm{C}$, $\mathrm{C} 10$ and $\mathrm{C} 11$, but the other propyl group involving $\mathrm{C} 12, \mathrm{C} 13$ and $\mathrm{C} 14$ was disordered (Figure 2), showing two tracks of weak electron density. We tried to refine this branch with split occupancy, but the electron density after refinement was not continuous. This result suggested that this branch adopted additional conformations and that each of the two tracks of weak electron density had much less than $50 \%$ occupancy. At this point, we decided to model this branch in one partially occupied conformation rather than all of the possible conformations.

Potential interactions between inhibitor 2 and N9 NA were assessed using Chimera [28]. In addition, potential hydrogen bonds as well as hydrophobic contacts were identified with HBPLUS [29] (see Table 3 for the geometric criteria). Favorable hydrophobic contacts were defined as non-bonded contacts between two carbon atoms at a distance of $\leq 4 \AA[30]$.

Structural studies of complexes between several benzoic acid leads and type B NA showed that these inhibitors were bound in the active site in a similar fashion to sialic acid [21,32], and the same is observed in the new structure (Figure 3). The carboxylate group of the inhibitor interacts with the guanidinium groups of arginine 
Table 2 X-ray diffraction data and refinement statistics

\begin{tabular}{|c|c|}
\hline A. X-ray Data & \\
\hline \multirow[t]{2}{*}{ X-ray Source } & SSRL \\
\hline & Beam line 7-1 \\
\hline Space Group & 1432 \\
\hline \multicolumn{2}{|l|}{ Cell Dimensions } \\
\hline$a=b=c(\AA)$ & 181.0 \\
\hline Asymmetric Unit & 1 monomer \\
\hline \multirow[t]{2}{*}{ Resolution Range ( $\AA$ ) } & $27.9-1.55$ \\
\hline & $(1.63-1.55)$ \\
\hline \multirow[t]{2}{*}{$R_{\text {merge }}$} & 0.067 \\
\hline & $(0.767)^{*}$ \\
\hline \multirow[t]{2}{*}{$R_{\text {p.im. }}{ }^{*}$} & 0.013 \\
\hline & $(0.25)$ \\
\hline \multirow[t]{2}{*}{ mean $(I / \sigma(I))$} & 29.4 \\
\hline & (3.8) \\
\hline \multirow[t]{2}{*}{ Multiplicity } & 24.7 \\
\hline & $(17.8)$ \\
\hline \multirow[t]{2}{*}{ No. Unique Reflections } & 72,486 \\
\hline & $(10,350)$ \\
\hline \multirow[t]{2}{*}{ Data Completeness (\%) } & 99.9 \\
\hline & $(99.1)$ \\
\hline \multicolumn{2}{|l|}{ B. Refinement } \\
\hline$R_{\text {work }}$ & 0.1267 \\
\hline$R_{\text {free }}$ & 0.1552 \\
\hline Bonds $(\AA)$ & 0.008 \\
\hline Angles $\left({ }^{\circ}\right)$ & 1.562 \\
\hline No. Amino Acids & 391 \\
\hline No. Glycan monomers & 15 \\
\hline No. D-glucose molecules & 4 \\
\hline No. Waters & 458 \\
\hline
\end{tabular}

(Statistics for the highest resolution shell are in parentheses).

${ }^{*} R_{\text {merge }}$ is high in the highest resolution shell due to the high multiplicity of the data. The $R_{p, i m}$ is independent of the data multiplicity and shows that the data in the highest shell have a reasonable discrepancy of $25 \%$. $R_{\text {p.i.m. }}$ is the precision indicating $R_{\text {merge }}$ [27].

residues at positions 118,292 , and 371 as seen in all NA substrate and inhibitor complexes and calculated to be energetically important [33]. A weak hydrogen bond is seen between Tyr406 and the carbonyl oxygen that interacts with Arg292. The pyrrolidine ring interacts with Arg152 by forming a hydrogen bond with its carbonyl oxygen (O15). The methylene group $\mathrm{C} 16$ lies in the hydrophobic pocket formed by Trp178 and Arg152. The methylene group $\mathrm{C} 17$ is involved in $\mathrm{C}-\mathrm{H}^{\cdots} \mathrm{O}$ bonds with Trp178 carbonyl O and Glu227 OE2. One of the hydroxyl methyl groups (O20) is hydrogen bonded to Glu277. Atom O20 also interacts with Glu276 through two water molecules, HOH553 and HOH612, by a chain of hydrogen bonds. The other hydroxyl methyl group (O19) was directed toward Trp178 O and Glu119 OE2. The one well-ordered propyl chain is anchored by hydrophobic contacts with Ile222, Ala246, and Arg224. The other propyl chain that is disordered is exposed to the solvent and therefore not contributing to binding energy because the one interaction seen (Table 3) is of low occupancy and at the upper limit of the distances considered as significant.

\section{Comparative analysis}

The model of 2 complexed with N9 NA was compared to sialic acid complexes with A/Tokyo/3/1967 N2 NA, PDB ID: 2BAT [35] and A/tern/Australia/G70c/75 N9 NA, PDB ID: 1MWE [26]. When our new structure was fitted with CCP4's SUPERPOSE [36] using the 11 active site residues, the root mean square deviations (RMSDs) were $0.7 \AA$ to $\mathrm{N} 2$ and $0.4 \AA$ to N9. The model gave a RMSD of $1.65 \AA$ when superposed on the complex of compound 1 with B/Lee/40 NA [21] using COOT secondary structure matching [37]. These small RMSDs suggest that replacing the sialic acid ligand with the inhibitor did not disturb the orientation of the active site residues of NA. The larger deviation between $\mathrm{N} 9$ and $\mathrm{B}$ NAs was expected given that there is less than $30 \%$ sequence identity. In all the above comparisons, most of the active site residues (Asn151, Arg152, Glu227, Arg371, Arg292 and Arg118-numbering as in the current complex) superposed well in the two molecules and a maximum shift of 0.2 to $0.5 \AA$ was observed. However, the side chain of Glu276 showed significant conformational change in the current complex when compared to NA-sialic acid or NA-zanamivir complexes. The two oxygen atoms OE1 and OE2 of Glu227 in the current complex moved toward the solvent and away from the active site by $1 \AA$. In this position, the carboxyl group interacted with NE of Arg224 and NH2 of His274. Hence, Glu276 did not form the direct hydrogen bonds with the inhibitor hydroxyl oxygen O20 analogous to those that Glu276 formed with the glycerol side chain of sialic acid and its transition state mimics. However, O20 of the inhibitor was linked to Glu276 through the water molecules $\mathrm{HOH} 552$ and $\mathrm{HOH} 611$. The $\mathrm{C} 14$ atom of the inhibitor is seen to make a hydrophobic contact with Glu276 but the low occupancy of the C12-C14 chain precludes a significant contribution to binding.

In the compound 1 complex with influenza B NA [21], the aliphatic chain forms van der Waals contacts with the side chains of Arg292, Asn294 and Glu275 while the hydroxymethyl groups interact with Glu117, Trp177 and Glu276. The rotation of the Glu276 side chain towards Arg224 observed in our complex was noted in the other structures where the inhibitor carries a hydrophobic side chain [21]. N1 NAs have additional flexibility compared to N9 in the 150 loop but binding of oseltamivir to wild-type N1 NA 


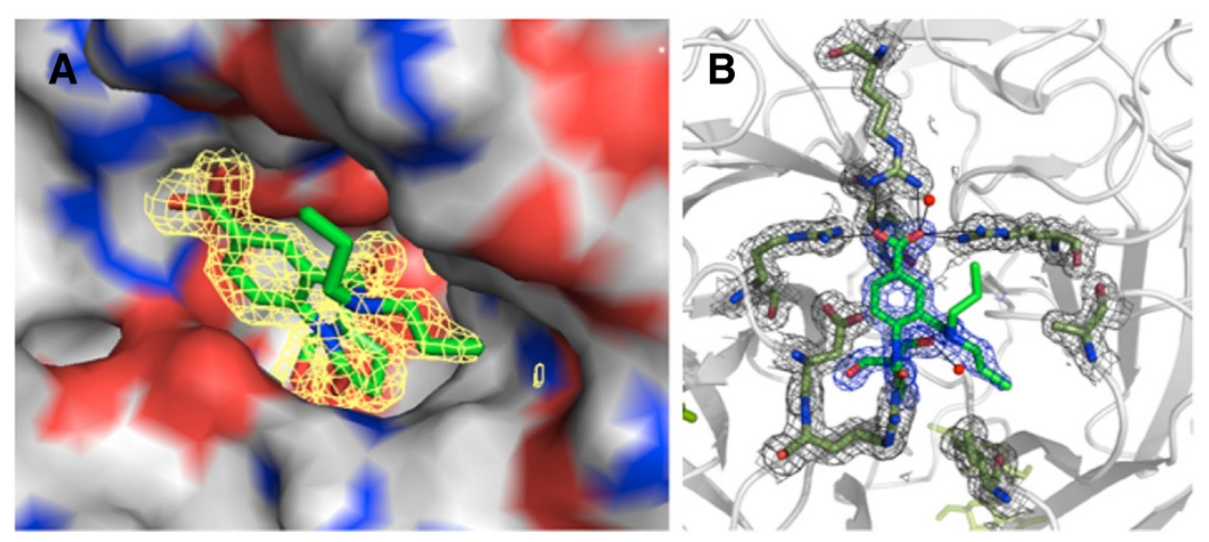

Figure 2 Electron density of inhibitor 2 in the active site. $A, 2 F_{\mathrm{o}}-F_{\mathrm{c}}$ map of inhibitor contoured at $1 \sigma$ shows inhibitor fitting snugly in the active site cavity; $B, 2 F_{\mathrm{o}}-F_{\mathrm{c}}$ map of inhibitor and interacting amino acids of the NA.

involves a conformational change in the side chain of Glu276 relative to the ligand free enzyme $[20,38]$ similar to that seen in N9 NAs.

We compared the NA and inhibitor contacts with previously reported benzoic acid inhibitor-NA structures using Chimera with the relatively stringent constraint of distance $\leq 3.5 \AA$ and including both polar and hydrophobic contacts. In the BANA 113-B NA complex [15] [39], 12 drug atom made 21 contacts $\leq 3.5 \AA$ with 10 amino acids of NA. In 1-B NA [21], 14 drug atoms make 23 contacts with 13 amino acids. Inhibitor 2 shows a small increase to 15 drug atoms making 28 contacts with 12 amino acids. The benzene ring of $\mathbf{2}$ is tilted by $8.9^{\circ}$ relative to compound 1 (Figure 4), increasing the number of contacts as was predicted in the design. However, one branch of the 3-heptyl group makes no significant contacts due to multiple conformations, which may be why the IC50 is no better than the previous compounds (Table 1).

Inhibitor 2 did not bind to the second sialic acid binding site observed in the structure of N9 complexed with sialic acid at low temperature [26] (PDB ID, 1MWE) or at room temperature (PDB ID, 2CML).

\section{Glycan structures}

When compared to the structure of the N9 mutant R292K (PDB ID, 2QWA) and the 1.4 $\AA$ resolution structures of native N9 in complex with other inhibitors (PDB IDs, 1F8D and1F8E), our structure has four additional sugar units in the glycan chains attached to the delta nitrogen atoms of Asn146 and Asn200 (N2 numbering) (Figure 5). At site 146, we found a second NAG residue and evidence for a $\beta$-D-mannose (BMA). At site 200, we found two additional mannose residues. One mannose (Man477) is bonded to the O6 oxygen of Man475G. The second mannose (Man476H) is linked to the O3 oxygen atom of Man475G, resulting in a GlcNAc $-\mathrm{Man}_{7}$ structure (Figure 5B). This glycan attached to Asn200 contacts 11 amino acids of the neighboring subunit when the tetramer is built by symmetry (Table 4 and Figure 5C). A high-mannose glycan at Asn200 is also present in N2 (A/ Tokyo/67) NA, where it interacts with the adjacent symmetry-related subunit at amino acids 391-394 and 453-455 (PDB ID, 1NN2). This glycan forms part of the Mem5 monoclonal antibody epitope on the 1998 N2 NA, and its interactions with the adjacent subunit are not disturbed by the presence of the antibody [41], suggesting the subunit interactions are energetically significant. In N9 there is experimental evidence that the glycan attached to Asn200 contributes to folding or oligomerization because the mutation N200L in N9 NA resulted in $80 \%$ reduction in enzyme activity although the expression level was the same as wild type [42]. In both N2 and N9 this subunit-spanning glycan is of the high mannose type, as is the glycan at 86 in N2 [43]. N6 NA (PDB ID $1 \mathrm{VOZ}$ ) crystallized with the tetramer as the asymmetric unit, so there is direct evidence for the inter-subunit interaction of the glycan attached to Asn200 in N6 NA. N1 NAs do not have a predicted glycosylation site at 200 , but a study of N1 NA assembly suggested that tetramerization requires high-mannose glycans. When all the glycans were processed to complex structures then the resulting dimers and monomers did not assemble into tetramers [44]. The PDB structures of N1 NA have no density for sugar residues, so it is not known if one of the other glycans is a high-mannose structure that spans the subunit interface in the same way as the glycan at Asn200 in N9, N2 and N6.

\section{Glucose molecules}

We used $49 \%(\mathrm{w} / \mathrm{v})$ glucose as cryoprotectant, and we observed four glucose molecules bound with greater than $50 \%$ occupancy in the structure of the inhibitor complex (Figure 4A). The glucose molecules are in a mix of alpha and beta configurations about the anomeric carbon atom. The O3 oxygen atom of Glc487 interacts with the ND2 
Table 3 Contacts observed between inhibitor 2 and N9 NA; 3LV is the residue name assigned to the inhibitor by the PDB and 488 is the residue number of the inhibitor

\begin{tabular}{|c|c|c|c|c|}
\hline Inhibitor atom & N9 atom & distance $(\AA ̊)$ & Angle(D-A-AA) ${ }^{\circ}$ & \\
\hline \multicolumn{5}{|c|}{ Potential hydrogen bonds ${ }^{1}$} \\
\hline 3LV488-O1 & Arg118-NH1 & 2.84 & 117.3 & \\
\hline 3LV488-O1 & Arg371-NH1 & 2.86 & 112.8 & \\
\hline 3LV488-O2 & Arg292-NH1 & 3.34 & 97.1 & \\
\hline 3LV488-O2 & Arg292-NH2 & 3.25 & 101.2 & \\
\hline 3LV488-O2 & Arg371-NH2 & 2.84 & 124.9 & \\
\hline \multirow[t]{2}{*}{ 3LV488-O2 } & $\mathrm{HOH}-747$ & 2.85 & & \\
\hline & $\mathrm{HOH}-747$ & 3.25 & & Asn347 OD1 \\
\hline 3LV488-O15 & Arg152- NH1 & 2.54 & 145.9 & \\
\hline \multirow[t]{2}{*}{ 3LV488-O20 } & $\mathrm{HOH}-612$ & 2.72 & & \\
\hline & $\mathrm{HOH}-612$ & $2.82,3.16$ & & Glu227 OE2, Thr225 O \\
\hline \multicolumn{5}{|c|}{ Van der Waals contacts ${ }^{2}(\leq 4.0 \AA)$} \\
\hline \multirow[t]{4}{*}{ 3LV488-C1 } & Tyr406-OH & 2.97 & 119.4 & \\
\hline & Arg371-NH1 & 3.70 & 96.2 & \\
\hline & Arg371-NH2 & 3.49 & 106.5 & \\
\hline & Tyr406-CZ & 3.83 & 42.5 & \\
\hline \multirow[t]{5}{*}{ 3LV488-O1 } & Tyr406-OH & 3.50 & 99.8 & \\
\hline & Arg118-CZ & 3.65 & 43.7 & \\
\hline & Arg118-NH2 & 3.56 & 83.0 & \\
\hline & Arg371-CZ & 3.59 & 47.2 & \\
\hline & Arg371-NH2 & 3.45 & 85.5 & \\
\hline 3LV488-C2 & Tyr406-OH & 2.80 & 136.4 & \\
\hline \multirow[t]{3}{*}{ 3LV488-O2 } & Tyr406-OH & 3.32 & $123.9^{3}$ & \\
\hline & Arg371-CZ & 3.76 & 38.3 & \\
\hline & Arg371-NH1 & 3.82 & 96.2 & \\
\hline 3LV488-C3 & Tyr406-OH & 3.19 & 161.8 & \\
\hline \multirow[t]{4}{*}{ 3LV488-C6 } & Glu119-OE2 & 3.39 & 119.6 & \\
\hline & Asp151-CG & 3.49 & 74.6 & \\
\hline & Asp151-OD1 & 3.37 & 84.4 & \\
\hline & Asp151-OD2 & 3.87 & 62.7 & \\
\hline \multirow[t]{5}{*}{ 3LV488-C7 } & Glu119-OE2 & 3.49 & 136.6 & \\
\hline & Asp151-OD1 & 3.52 & 97.7 & \\
\hline & Asp151-CG & 3.89 & 63.8 & \\
\hline & Tyr406-OH & 3.23 & 118.0 & \\
\hline & Arg118-NH1 & 3.63 & 113.4 & \\
\hline 3LV488-C10 & Ile222-CD1 & 3.63 & 122.8 & \\
\hline \multirow[t]{6}{*}{ 3LV488-C11 } & Ala246-CB & 3.90 & 115.5 & \\
\hline & Ile222-CD1 & 3.54 & 105.5 & \\
\hline & Ile222-CB & 3.88 & 87.6 & \\
\hline & Arg224-CZ & 3.43 & 79.4 & \\
\hline & Arg224-NE & 3.78 & 64.8 & \\
\hline & Arg224-NH1 & 3.44 & 78.4 & \\
\hline
\end{tabular}


Table 3 Contacts observed between inhibitor 2 and N9 NA; 3 LV is the residue name assigned to the inhibitor by the PDB and 488 is the residue number of the inhibitor (Continued)

\begin{tabular}{|c|c|c|c|}
\hline & Arg224-NH2 & 3.81 & 63.4 \\
\hline & $\mathrm{HOH}-945$ & 2.82 & - \\
\hline 3LV488-C14 & Arg292-NH2 ${ }^{4}$ & 3.50 & 140.0 \\
\hline \multirow[t]{4}{*}{ 3LV488-O15 } & Asp151-CB & 3.59 & 100.5 \\
\hline & Arg152-CG & 3.84 & 73.6 \\
\hline & Arg152-CD & 3.71 & 83.0 \\
\hline & Arg152-CZ & 3.70 & 22.6 \\
\hline \multirow[t]{5}{*}{ 3LV488-C16 } & Trp178-CE3 & 3.59 & 91.2 \\
\hline & Trp178-CZ3 & 3.88 & 67.8 \\
\hline & Trp178-O & 3.78 & 135.7 \\
\hline & Arg152-CD & 3.73 & 78.1 \\
\hline & Arg152-CG & 3.73 & 78.1 \\
\hline \multirow[t]{2}{*}{ 3LV488-C17 } & Trp178-O & 3.43 & 139.6 \\
\hline & Glu227-OE2 & 3.62 & 135.8 \\
\hline \multirow[t]{2}{*}{ 3LV488-C19 } & Trp178-O & 3.19 & 167.7 \\
\hline & Glu119-CD & 3.88 & 85.8 \\
\hline \multirow[t]{4}{*}{ 3LV488-O19 } & Asp151-O & 3.26 & 116.7 \\
\hline & Asp151-CB & 3.73 & 97.2 \\
\hline & Arg156-NH1 & 3.68 & 139.0 \\
\hline & Trp178-O & 2.82 & 162.5 \\
\hline \multirow[t]{4}{*}{ 3LV488-C20 } & Glu227-CG & 3.88 & 74.3 \\
\hline & Glu227-CD & 3.77 & 55.7 \\
\hline & Glu227-OE2 & 3.23 & 105.4 \\
\hline & Glu277-OE2 & 3.34 & 140.2 \\
\hline \multirow[t]{4}{*}{ 3LV488-O20 } & Glu227-OE2 & 3.68 & 115.2 \\
\hline & Glu277-CG & 3.80 & 70.4 \\
\hline & Glu277-CD & 3.59 & 31.9 \\
\hline & Glu277-OE2 & 2.61 & 133.4 \\
\hline \multicolumn{4}{|c|}{ Water bridge - inhibitor - Glu276 } \\
\hline 3LV488-O20 & $\mathrm{HOH}-612$ & 2.72 & \\
\hline $612-\mathrm{HOH}$ & $553-\mathrm{HOH}$ & 2.64 & \\
\hline $553-\mathrm{HOH}$ & Glu276-OE1 & 2.82 & 142.2 \\
\hline \multicolumn{4}{|c|}{ Glu276 interactions } \\
\hline Glu276-OE1 & Arg224-NE & 2.72 & 114.2 \\
\hline Glu276-OE2 & Arg224-NE & 3.50 & 83.2 \\
\hline Glu276-OE2 & Arg224-NH2 & 2.81 & 115.9 \\
\hline Glu276-OE2 & His274-NE2 & 2.73 & 119.7 \\
\hline
\end{tabular}

TPotential hydrogen bonds were identified using HBPLUS [29]. Potential hydrogen bonds were interactions between donor and acceptor atoms that met the following geometric requirements: (i) a donor acceptor distance (D-A) $<3.5 \AA$ and a hydrogen acceptor distance (H-A) $<2.5 \AA$ and (ii) a donor-acceptor-acceptor antecedent angle (D-A-AA), of $>90^{\circ}$ [31]. ${ }^{2}$ Favorable hydrophobic contacts were defined as non-bonded contacts between two carbon atoms at a distance of $\leq 4 \AA$ [28].

${ }^{3}$ Freely rotating hydroxyl hydrogen atoms had the potential to form hydrogen bonds with two alternative acceptor atoms. Only the more probable acceptor atom at the shorter distance from the hydrogen atom was reported by HBPLUS.

${ }^{4}$ There were several conformations of the propyl chain C12-C14. We used the best, but it is of low occupancy so the interactions involving C14 contribute very little.

nitrogen atom of Arg141 through a hydrogen bond. O3 and $\mathrm{O} 1$ of Glc486 forms hydrogen bonds with NE2 of Gln315 and ND2 of Asn338 respectively.

\section{Conclusions}

Our aim in designing inhibitor 2 was to extend the substituent at C4, corresponding to $\mathrm{C} 6$ of sialic acid, to (i) increase 

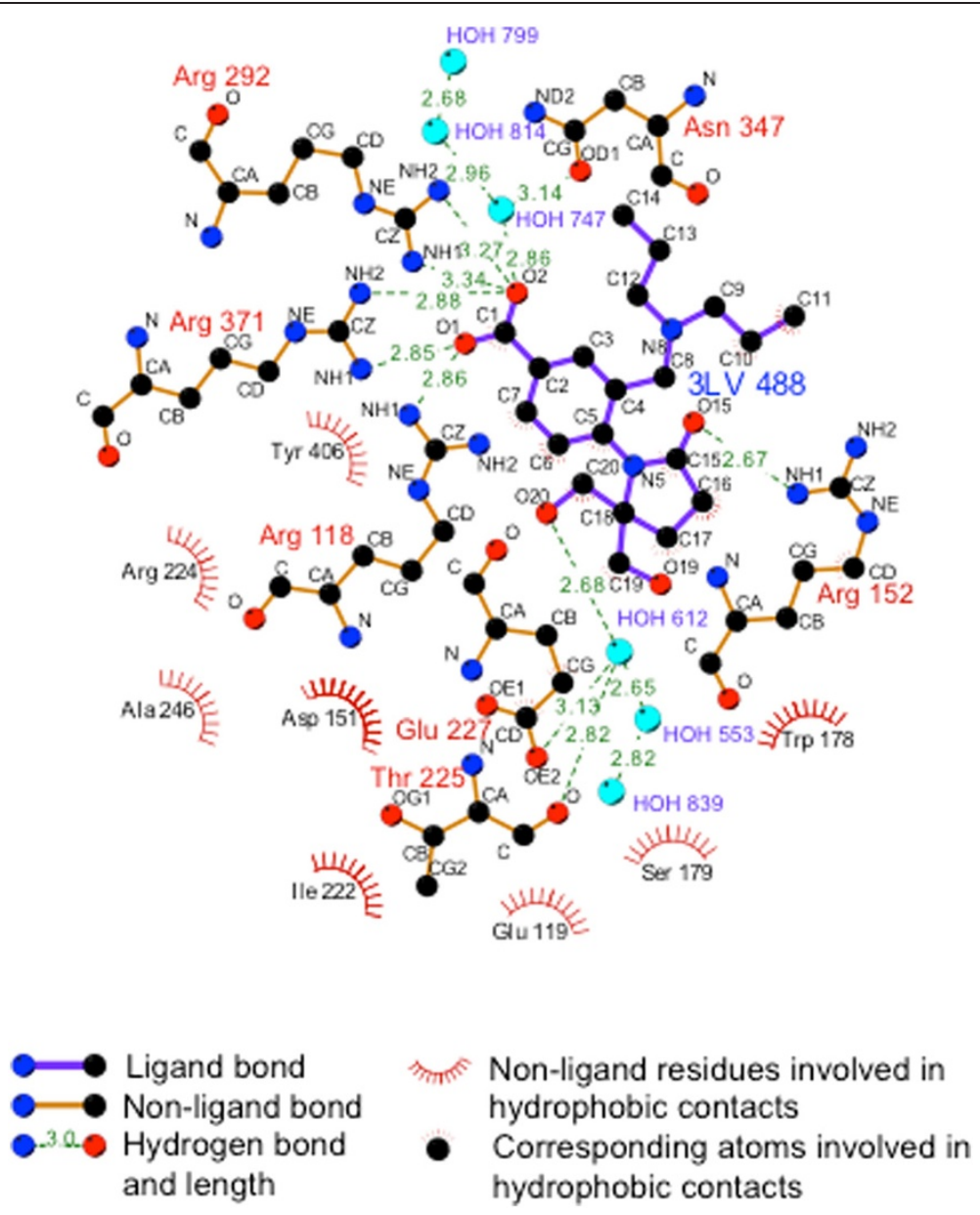

Figure 3 Interactions between compound 2 and NA. Ligplot + [34] diagram showing interactions.

the contact surface in the C6-subsite and (ii) force the benzene ring to tilt to maximize these interactions while retaining the interactions of the carboxylate and the pyrolidinone substituents. The crystal structure at $1.55 \AA$ shows that we were partially successful in that the ring in $\mathbf{2}$ is tilted relative to compound $\mathbf{1}$ (Figure 4) and the overall number of contacts is increased. The $\mathrm{IC}_{50}$ did not decrease, and the reason became clear when we solved the crystal structure. The second propyl group is not making any contacts but is freely waving above the surface of the NA. Future design efforts will include unequal branches that may be accommodated in the C6-subsite.

The N9 NA that was crystallized for this experiment was purified from virus grown in embryonated chicken eggs, so it contains a full complement of vertebrate processed N-linked glycans, in contrast to NA expressed in insect cells which has truncated glycan structures. We therefore refined the glycans as far as we could see electron density. Most glycans are flexible and only the first few sugars are seen, but the glycan attached to Asn200 is well resolved due to interaction with the adjacent subunit and is seen to contain GlcNAc $\mathrm{Man}_{7}$. Mutation data suggests this glycan plays a role in stabilizing the NA tetramer, which is a significant property because, for

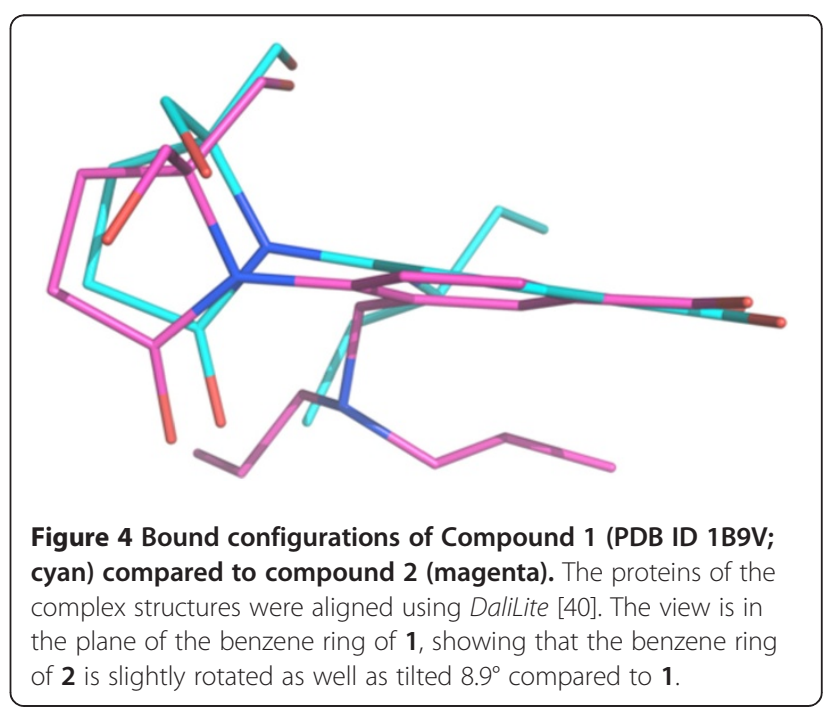



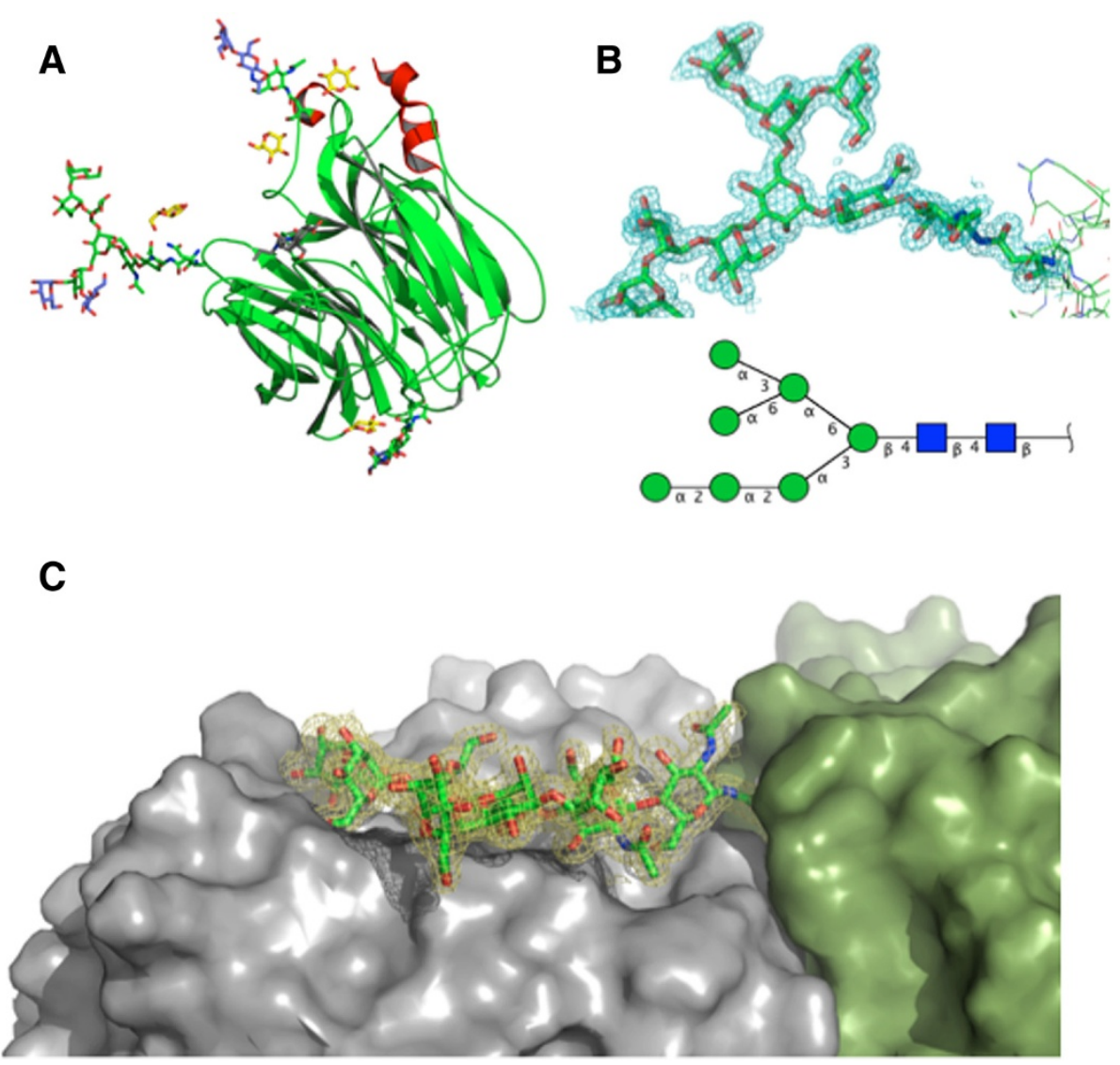

Figure 5 A. Carbohydrates in the N9-compound 2 model at 1.55 Å resolution. Carbon atoms are green for N9 NA and the N-linked glycans attached at positions 86, 146 and 200 that are in common with other structures. Mannose residues not previously seen in N9 structures (blue) and the four glucose molecules from the cryoprotectant (yellow) are shown. Compound $\mathbf{2}$ is colored gray. B. Electron density $\left(2 F_{\mathrm{o}}-F_{\mathrm{c}}\right.$ at $\left.1 \sigma\right)$ for the glycan at Asn200. Also shown is the cartoon of the glycan structure using standard Consortium for Functional Glycomics symbols (green circle, mannose; blue square, N-acetylglucosamine) made with Glycan Builder [45]. C. Interaction of the glycan attached to Asn200 (green) with the neighboring subunit (gray) built by applying crystallographic symmetry.

reasons that are not understood [9], only the NA tetramer has enzymatic activity.

\section{Materials and methods}

\section{Inhibitor synthesis}

The synthesis, purification and evaluation of the in vitro inhibitory activity of 2 \{4-[2,2-bis(hydroxymethyl)-5-oxopyrrolidin-1-yl]-3-[(dipropylamino)methyl]benzoic acid\} will be described elsewhere [23].

\section{Protein preparation and crystallization}

The reassortant virus $\mathrm{A} / \mathrm{NWS} / 33_{\mathrm{H}^{-}} \mathrm{A} / \mathrm{tern} /$ Australia/G70C/ $75_{\mathrm{N}}$ (H1N9) was grown and purified as previously described [25]. The purified virus was digested with Pronase (Sigma) at a concentration of $6 \mathrm{mg} / \mathrm{ml}$ at $37 \mathrm{C}$ for 16 hours. The cores were removed by centrifugation, and the released heads were pooled, concentrated, and purified by gel filtration using FPLC [41]. The purified protein was concentrated to $10 \mathrm{mg} /$ $\mathrm{ml}$. Single crystals of N9 NA were grown by vapor diffusion using the hanging drop method containing equal volumes of $\mathrm{N} 9$ and the reservoir solution of $1.9 \mathrm{M}$ potassium phosphate buffer $\mathrm{pH} 6.9$ [8].

Inhibitor soaking, cryoprotection and X-ray data collection The successful flash cooling of large $(0.2-0.4 \mathrm{~mm}$ per edge) cubic crystals in liquid nitrogen required serial equilibration by vapor diffusion. The estimated osmolality for $1.9 \mathrm{MK}$ phosphate buffer ( $\mathrm{pH}$ 6.9) in the reservoir solution was matched to that of glucose using standard tables $[46,47]$. Crystals were placed over wells with $200 \mathrm{mM} \mathrm{K}$-phosphate buffer ( $\mathrm{pH}$ 6.9) containing $45 \mathrm{~g} / 100 \mathrm{ml}(45 \% \mathrm{w} / \mathrm{v}$ ) glucose for 6 hours to two days and then placed over a well with $46 \%$ glucose. This procedure was repeated until the crystals were over wells that contained $49 \%$ glucose. After the final equilibration, the crystals were soaked in $49 \%$ glucose and $200 \mathrm{mM}$ K-phosphate with $25 \mathrm{mM}$ inhibitor for 5 minutes before they were mounted in a rayon cryoloop and vitrified at $100 \mathrm{~K}$ in a nitrogen cold-stream. 


\begin{tabular}{|c|c|c|c|c|}
\hline Sugar & Sugar atom & Amino acid & Atom & Distance $(\AA ̊)$ \\
\hline \multirow[t]{4}{*}{ NAG469A } & O5 & G454 & $C A$ & 3.39 \\
\hline & & Q455 & $\mathrm{N}$ & 3.35 \\
\hline & $\mathrm{C} 6$ & $\llcorner 453$ & O & 3.43 \\
\hline & O6 & Q455 & $C G$ & 3.25 \\
\hline \multirow[t]{2}{*}{ NAG 470B } & $\mathrm{O} 3$ & G394 & $\mathrm{N}$ & 2.96 \\
\hline & $\mathrm{C3}$ & G394 & $\mathrm{O}$ & 3.16 \\
\hline BMA 471 C & $\mathrm{O} 2$ & G394 & CA & 3.21 \\
\hline \multirow[t]{4}{*}{ MAN 472D } & $\mathrm{O} 3$ & R364 & $\mathrm{NH} 2$ & 3.02 \\
\hline & $\mathrm{C} 3$ & E375 & OE1 & 3.39 \\
\hline & $\mathrm{O} 3$ & E375 & OE2 & 2.58 \\
\hline & $\mathrm{O} 4$ & E375 & OE1 & 2.75 \\
\hline \multirow[t]{3}{*}{ MAN 473E } & O5 & R364 & $\mathrm{NH} 2$ & 3.15 \\
\hline & O6 & K389 & NZ & 2.82 \\
\hline & O6 & D330 & OD2 & 2.54 \\
\hline \multirow[t]{6}{*}{ MAN $473 \mathrm{~F}$} & $\mathrm{O} 2$ & N329 & OD1 & 3.10 \\
\hline & $\mathrm{O} 3$ & D330 & N & 2.93 \\
\hline & $\mathrm{O} 3$ & N329 & OD1 & 2.74 \\
\hline & $\mathrm{O} 3$ & N329 & $C A$ & 2.99 \\
\hline & $\mathrm{O} 4$ & R327 & $\mathrm{NH} 2$ & 3.11 \\
\hline & O6 & 1366 & 0 & 2.75 \\
\hline
\end{tabular}

$\mathrm{X}$-ray data were collected at $100 \mathrm{~K}$ at SSRL beam line 7-1 on an ADSC Quantum 315 CCD detector using monochromatic radiation with a wavelength of $0.9537 \AA$. The X-ray data were collected at distances of 300 and $220 \mathrm{~mm}$ with oscillation angles of $0.5^{\circ}$ and $0.35^{\circ}$ respectively and with $2 \mathrm{~s}$ exposures at both distances.

The X-ray data were indexed and integrated with $X D S$ [48]. The integrated intensities were scaled and merged with the CCP4 [49] program SCALA [50] and converted to structure factors with TRUNCATE [51].

\section{Structure determination and analysis}

The structure was solved by molecular substitution. The $1.7 \AA$ crystal structure of the R292K mutant of tern N9 influenza virus NA [24] (2QWA) without the glycans or solvent molecules was used as the starting model in rigid body and coordinate refinement in REFMAC [52]. Refinement was continued using PHENIX [53] together with iterative rounds of model rebuilding using the molecular graphic package COOT [37]. The PRODRG webserver [54] was used to build the initial coordinates and stereochemical restraints of inhibitor 2 . The restraints for three $\beta$-D-mannose monomers (BMA) were taken from the REFMAC library [34]. Riding hydrogen atoms were added with the program REDUCE [55].
The structure was analyzed with HBPLUS [29] and Chimera [28]. The figures were made using $P y M O L$ (Schrödinger LLC) and Liglot+ [56].

\section{Accession codes}

The atomic coordinates and structure factors were deposited in the protein data bank under accession code 4DGR for the inhibitor complex.

Competing interests

The authors declare that they have no competing interests.

\section{Acknowledgements}

We thank Dr Tzanko Doukov for help with data collection at Stanford Synchrotron Radiation Lightsource (SSRL) beam line 7-1. The Structural Molecular Biology Program at SSRL is supported by DOE-OBER, NIH-NCRR (P41RR001209), and NIH-NIGMS. Some refinements with PHENIX were run remotely at the OU Supercomputing Center for Education \& Research (OSCER) at the University of Oklahoma. We thank OSCER Director Dr Henry Neeman and Joshua Alexander for valuable technical assistance and Shelly Gulati for growth and purification of virus and determination of the IC50. This work was supported by NIAID grant Al 062950 to WJB.

\section{Authors' contributions}

WJB, BHMM and GMA designed the research, analyzed the results and wrote the manuscript. WJB, ESJ and GK designed and synthesized the inhibitor and GMA tested it. BHMM and LV crystallized the complex, solved and refined the structure, analyzed the structure and drafted the manuscript. All authors read and approved the final manuscript.

Received: 24 February 2012 Accepted: 13 April 2012

Published: 6 May 2012

\section{References}

1. Palese P, Tobita K, Ueda M, Compans RW: Characterization of temperature-sensitive influenza virus mutants defective in neuraminidase. Virology 1974, 61:397-410.

2. Liu C, Eichelberger MC, Compans RW, Air GM: Influenza type A virus neuraminidase does not play a role in viral entry, replication, assembly, or budding. J Virol 1995, 69(2):1099-1106.

3. Webster RG, Govorkova EA: H5N1 influenza-continuing evolution and spread. New Engl J Med 2006, 355(21):2174-2177.

4. Rota P: Cocirculation of two distinct evolutionary lineages of influenza type B virus since 1983. Virology 1990, 175(1):59-68.

5. Air GM, Brouillette WJ: Influenza virus antiviral targets. In Antiviral Res. Edited by LaFemina R. Washington, DC: ASM Press; 2009:187-207.

6. Colman PM, Varghese JN, Laver WG: Structure of the catalytic and antigenic sites in influenza virus neuraminidase. Nature 1983, 303:41-44.

7. Burmeister WP, Ruigrok RW, Cusack S: The 2.2 A resolution crystal structure of influenza B neuraminidase and its complex with sialic acid. EMBO J 1992, 11(1):49-56.

8. Bossart-Whitaker P, Carson M, Babu YS, Smith CD, Laver WG, Air GM Three-dimensional structure of influenza A N9 neuraminidase and its complex with the inhibitor 2-deoxy 2,3-dehydro-N-acetyl neuraminic acid. J Mol Biol 1993, 232(4):1069-1083.

9. Air GM: Influenza neuraminidase. Influenza Other Respir Viruses 2011, eprint Nov 16, DOI:10.1111/j.1750-2659.2011.00304.x.

10. von Itzstein M, Wu WY, Kok GB, Pegg MS, Dyason JC, Jin B, Van Phan T, Smythe ML, White HF, Oliver SW, et al: Rational design of potent sialidase-based inhibitors of influenza virus replication. Nature 1993, 363 (6428):418-423.

11. Kim CU, Lew W, Williams MA, Liu H, Zhang L, Swaminathan S, Bischofberger $N$, Chen MS, Mendel DB, Tai CY, et al: Influenza neuraminidase inhibitors possessing a novel hydrophobic interaction in the enzyme active site: design, synthesis, and structural analysis of carbocyclic sialic acid analogues with potent anti-influenza activity. J Am Chem Soc 1997, 119 (4):681-690

12. Brouillette WJ, Atigadda VR, Luo M, Air GM, Babu YS, Bantia S: Design of benzoic acid inhibitors of influenza neuraminidase containing a cyclic 
substitution for the N-acetyl grouping. Bioorg Med Chem Lett 1999, 9 (14):1901-1906.

13. Kim CU, Lew W, Williams MA, Wu H, Zhang L, Chen X, Escarpe PA, Mendel DB, Laver WG, Stevens RC: Structure-activity relationship studies of novel carbocyclic influenza neuraminidase inhibitors. J Med Chem 1998, 41 (14):2451-2460

14. Babu YS, Chand P, Bantia S, Kotian P, Dehghani A, El-Kattan Y, Lin TH, Hutchison TL, Elliott AJ, Parker CD, et al: BCX-1812 (RWJ-270201): discovery of a novel, highly potent, orally active, and selective influenza neuraminidase inhibitor through structure-based drug design. J Med Chem 2000, 43(19):3482-3486.

15. Jedrzejas MJ, Singh S, Brouillette WJ, Air GM, Luo M: A strategy for theoretical binding constant, $\mathrm{Ki}$, calculations for neuraminidase aromatic inhibitors designed on the basis of the active site structure of influenza virus neuraminidase. Proteins 1995, 23(2):264-277.

16. Singh S, Jedrzejas MJ, Air GM, Luo M, Laver WG, Brouillette WJ: Structurebased inhibitors of influenza virus sialidase. A benzoic acid lead with novel interaction. J Med Chem 1995, 38(17):3217-3225.

17. Brouillette WJ, Bajpai SN, Ali SM, Velu SE, Atigadda VR, Lommer BS, Finley JB, Luo M, Air GM: Pyrrolidinobenzoic acid inhibitors of influenza virus neuraminidase: modifications of essential pyrrolidinone ring substituents. Bioorg Med Chem 2003, 11(13):2739-2749.

18. Atigadda VR, Brouillette WJ, Duarte F, Ali SM, Babu YS, Bantia S, Chand P, Chu N, Montgomery JA, Walsh DA, et al: Potent inhibition of influenza sialidase by a benzoic acid containing a 2-pyrrolidinone substituent. J Med Chem 1999, 42(13):2332-2343.

19. Smith PW, Robinson JE, Evans DN, Sollis SL, Howes PD, Trivedi N, Bethell RC: Sialidase inhibitors related to zanamivir: synthesis and biological evaluation of $4 \mathrm{H}$-pyran 6-ether and ketone. Bioorg Med Chem Lett 1999, 9 (4):601-604

20. Russell RJ, Haire LF, Stevens DJ, Collins PJ, Lin YP, Blackburn GM, Hay AJ, Gamblin SJ, Skehel JJ: The structure of H5N1 avian influenza neuraminidase suggests new opportunities for drug design. Nature 2006 , 443(7107):45-49.

21. Finley JB, Atigadda VR, Duarte F, Zhao JJ, Brouillette WJ, Air GM, Luo M: Novel aromatic inhibitors of influenza virus neuraminidase make selective interactions with conserved residues and water molecules in the active site. J Mol Biol 1999, 293(5):1107-1119.

22. Rarey M, Kramer B, Lengauer T, Klebe G: A fast flexible docking method using an incremental construction algorithm. J Mol Biol 1996, 261(3):470489 .

23. Kolavi G, Li Y, Johnson ES, Gulati S, Air GM, Brouillette WJ: in preparation

24. Varghese JN, Smith PW, Sollis SL, Blick TJ, Sahasrabudhe A, McKimmBreschkin JL, Colman PM: Drug design against a shifting target: a structural basis for resistance to inhibitors in a variant of influenza virus neuraminidase. Structure 1998, 6(6):735-746.

25. Laver WG, Colman PM, Webster RG, Hinshaw VS, Air GM: Influenza virus neuraminidase with hemagglutinin activity. Virology 1984, 137:314-323.

26. Varghese JN, Colman PM, van Donkelaar A, Blick TJ, Sahasrabudhe A, McKimm-Breschkin JL: Structural evidence for a second sialic acid binding site in avian influenza virus neuraminidases. Proc Natl Acad Sci USA 1997, 94(22):11808-11812.

27. Weiss MS: Global indicators of X-ray data quality. J App/ Cryst 2001 34:130-135.

28. Pettersen EF, Goddard TD, Huang CC, Couch GS, Greenblatt DM, Meng EC, Ferrin TE: UCSF Chimera-a visualization system for exploratory research and analysis. J Comput Chem 2004, 25(13):1605-1612

29. McDonald I, Thornton J: Satisfying hydrogen bonding potential in proteins. J Mol Biol 1994, 238:777-793.

30. Copeland RA: Enzymes: A Practical Introduction to Structure, Mechanism, and Data Analysis. 2nd edition. New York: Wiley-VCH; 2000.

31. Baker EN, Hubbard RE: Hydrogen bonding in globular proteins. Prog Biophys Mol Biol 1984, 44(2):97-179.

32. Lommer BS, Ali SM, Bajpai SN, Brouillette WJ, Air GM, Luo M: A benzoic acid inhibitor induces a novel conformational change in the active site of Influenza B virus neuraminidase. Acta Crystallogr D Biol Crystallogr 2004, 60 (Pt 6):1017-1023.

33. Wang $T$, Wade RC: Comparative binding energy (COMBINE) analysis of influenza neuraminidase-inhibitor complexes. J Med Chem 2001, 44 (6):961-971.
34. Vagin AA, Steiner RA, Lebedev AA, Potterton L, McNicholas S, Long F, Murshudov GN: REFMAC5 dictionary: organization of prior chemical knowledge and guidelines for its use. Acta Crystallogr D Biol Crystallogr 2004, 60(Pt 12 Pt 1):2184-2195.

35. Varghese JN, McKimm-Breschkin JL, Caldwell JB, Kortt AA, Colman PM: The structure of the complex between influenza virus neuraminidase and sialic acid, the viral receptor. Proteins 1992, 14(3):327-332.

36. Krissinel $E$, Henrick K: Secondary-structure matching (SSM), a new tool for fast protein structure alignment in three dimensions. Acta Crystallogr D Biol Crystallogr 2004, 60(Pt 12 Pt 1):2256-2268.

37. Emsley P, Lohkamp B, Scott WG, Cowtan K: Features and development of Coot. Acta Crystallogr D Biol Crystallogr 2010, 66(Pt 4):486-501.

38. Collins PJ, Haire LF, Lin YP, Liu J, Russell RJ, Walker PA, Skehel JJ, Martin SR, Hay AJ, Gamblin SJ: Crystal structures of oseltamivir-resistant influenza virus neuraminidase mutants. Nature 2008, 453(7199):1258-1261.

39. Jedrzejas MJ, Singh S, Brouillette WJ, Laver WG, Air GM, Luo M: Structures of aromatic inhibitors of influenza virus neuraminidase. Biochemistry 1995, 34:3144-3151

40. Holm L, Park J: DaliLite workbench for protein structure comparison. Bioinformatics 2000, 16(6):566-567.

41. Venkatramani L, Bochkareva E, Lee JT, Gulati U, Graeme Laver W, Bochkarev A, Air GM: An epidemiologically significant epitope of a 1998 human influenza virus neuraminidase forms a highly hydrated interface in the NA-antibody complex. J Mol Biol 2006, 356(3):651-663. and cover picture.

42. Lee JT, Air GM: Contacts between influenza N9 neuraminidase and monoclonal antibody NC10. Virology 2002, 300:255-268.

43. Ward CW, Murray JM, Roxburgh CM, Jackson DC: Chemical and antigenic characterization of the carbohydrate side chains of an Asian (N2) influenza virus neuraminidase. Virology 1983, 126(1):370-375.

44. Wu Z, Ethen C, Hickey G, Jiang W: Active 1918 pandemic flu viral neuraminidase has distinct $\mathrm{N}$-glycan profile and is resistant to trypsin digestion. Biochem Biophys Res Commun 2009, 379:749-753.

45. Ceroni A, Dell A, Haslam S: The GlycanBuilder: a fast, intuitive and flexible software tool for building and displaying glycan structures. Source Code Biol Med 2007, 2:3.

46. Garman E: Cool data: quantity AND quality. Acta Crystallogr D Biol Crystallogr 1999, 55(Pt 10):1641-1653.

47. Weast RC (ed.): Handbook of Chemistry and Physics, 69th edn. Boca Raton, FL: CRC PRess; 1988.

48. Kabsch W: XDS. Acta Crystallogr D Biol Crystallogr 2010, 66(2):125-132.

49. Winn MD, Ballard CC, Cowtan KD, Dodson EJ, Emsley P, Evans PR, Keegan RM, Krissinel EB, Leslie AG, McCoy A, et al: Overview of the CCP4 suite and current developments. Acta Crystallogr D Biol Crystallogr 2011, 67(Pt 4):235242.

50. Evans P: Scaling and assessment of data quality. Acta Crystallogr D Biol Crystallogr 2006, 62(Pt 1):72-82.

51. French $\mathrm{S}$, Wilson $\mathrm{K}$ : On the treatment of negative intensity observations. Acta Crystallogr A 1978, 34:517-525

52. Murshudov GN, Vagin AA, Dodson EJ: Refinement of macromolecular structures by the maximum-likelihood method. Acta Crystallogr D Biol Crystallogr 1997, 53(Pt 3):240-255.

53. Adams PD, Afonine PV, Bunkoczi G, Chen VB, Davis IW, Echols N, Headd JJ, Hung LW, Kapral GJ, Grosse-Kunstleve RW, et al: PHENIX: a comprehensive Python-based system for macromolecular structure solution. Acta Crystallogr D Biol Crystallogr 2010, 66(Pt 2):213-221.

54. Schuttelkopf AW, van Aalten DM: PRODRG: a tool for high-throughput crystallography of protein-ligand complexes. Acta Crystallogr D Biol Crystallogr 2004, 60(Pt 8):1355-1363.

55. Word JM, Lovell SC, Richardson JS, Richardson DC: Asparagine and glutamine: using hydrogen atom contacts in the choice of side-chain amide orientation. J Mol Biol 1999, 285(4):1735-1747.

56. Laskowski, RA, Swindells, MB: LigPlot+: multiple ligand-protein interaction diagrams for drug discovery. J Chem Inf Model 2011, 51(10):2778-2786.

doi:10.1186/1472-6807-12-7

Cite this article as: Venkatramani et al:: Crystal structure of a new benzoic acid inhibitor of influenza neuraminidase bound with a new tilt induced by overpacking subsite C6. BMC Structural Biology 2012 12:7. 